\title{
Analysis of minor hysteresis loops in plastically deformed low carbon steel
}

\author{
S. Takahashi, ${ }^{\text {a) }}$ L. Zhang, S. Kobayashi, Y. Kamada, H. Kikuchi, and K. Ara \\ NDE and Science Research Center, Faculty of Engineering, Iwate University, Morioka 020-8551, Japan
}

(Received 7 July 2004; accepted 17 June 2005; published online 11 August 2005)

We have measured minor hysteresis loops with increasing magnetic-field amplitude $H_{a}$ step by step and have analyzed them in connection with lattice defects such as dislocations in deformed low carbon steel. We define several magnetic parameters in the minor loops: pseudocoercive force $H_{C}{ }^{*}$, pseudoremanence $M_{R}^{*}$, pseudosusceptibility at pseudocoercive force $\chi_{H}{ }^{*}$, pseudohysteresis loss $W_{F}{ }^{*}$, and pseudoremanence work $W_{R}{ }^{*}$. We find several simple relations between the pseudomagnetic properties, namely, $M_{R}{ }^{*} / M_{a}{ }^{*}=9 / 10$ and $3 / 5$, and $W_{R}{ }^{*} / W_{F}{ }^{*}=1 / 6$ and $1 / 8$, before and after plastic deformation, respectively. These relations are due to the similarity of minor loops. Six magnetic coefficients that are sensitive to lattice defects and are independent of $H_{a}$, as well as of the magnetic field, are obtained from the pseudomagnetic properties. These coefficients are effective parameters for nondestructive evaluation of degradation before the initiation of cracking. The minor-loop method has several advantages for nondestructive evaluation compared with the traditional method using major loops. The coefficients can give us much information about lattice defects with high accuracy. The measurements can be done at low magnetic fields less than $20 \mathrm{Oe}$ and the $H_{a}$ step does not necessarily have to be fine to obtain detailed information because of the similarity of minor loops. (C) 2005 American Institute of Physics. [DOI: 10.1063/1.1999853]

\section{INTRODUCTION}

The relationship between magnetism and lattice defects in ferromagnetic metals was studied by the Stuttgart group at the Max-Planck Institute four decades ago. ${ }^{1-3}$ The sensitive properties of a magnetic structure are represented by the dislocation density $\rho$ and its distribution; the coercive force $H_{C}$ increases in proportion to the square root of $\rho$, and the initial susceptibility $\chi_{i}$ decreases in inverse of the square root of $\rho$. The relationship was experimentally confirmed in $\mathrm{Ni}, \mathrm{Co}$, and $\mathrm{Fe}$ single crystals. These magnetic properties obtained from major hysteresis loops contain information on domainwall potential. Systematic study of this relationship has been summarized by Kronmüller and Fähnle. ${ }^{4}$

Recently, we found a method of analyzing minor hysteresis loops for Fe single crystals, where several pseudomagnetic properties were introduced, ${ }^{5,6}$ pseudocoercive force $H_{C}{ }^{*}$, pseudoremanence $M_{R}{ }^{*}$, pseudohysteresis loss $W_{F}{ }^{*}$, pseudoremanence work $W_{R}$, and three pseudosusceptibilities, $\chi_{R}{ }^{*}, \chi_{H}{ }^{*}$, and $\chi_{a}{ }^{*}$. These susceptibilities represent $180^{\circ}$ wall mobility at each point of the potential. We can obtain information on the potential energy for domain-wall displacement from these pseudomagnetic properties. The information about the potential obtained from the minor loops is more precise and detailed than the magnetostructure sensitive properties of the major loops. The potential energy depends on lattice defects such as grain boundaries, vacancies, interstitials, impurity atoms, voids, and dislocations. ${ }^{7}$

Our interest is the extension of the minor-loop method developed for Fe metals and alloys to A533B steel with plastic deformation. A533B steel is low carbon steel $(0.18$ wt $\% \mathrm{C}, 0.15$ wt $\% \mathrm{Si}, 150$ wt $\% \mathrm{Mn}, 0.03$ wt $\% \mathrm{Cu}$, $0.66 \mathrm{wt} \% \mathrm{Ni}, 0.56 \mathrm{wt} \% \mathrm{Mo}$, balance Fe), and is used in the

${ }^{a)}$ Electronic mail: seiki.t@iwate-u.ac.jp pressure vessel of nuclear reactors. Nondestructive evaluation of degradation is the most important and urgent theme for the pressure vessel, since the degradation of the pressure vessel determines the lifetime of the nuclear reactor. The purpose of the present study is to examine the relation between magnetic properties of minor loops and dislocations in A533B steel. The present method based on minor hysteresis loops is useful for nondestructive evaluation and is more advantageous for nondestructive tests compared with the traditional major-loop method where coercive force, initial susceptibility, and Rayleigh constant are determined. For example, the magnetic-field amplitude of minor loops can be small enough to obtain the detailed information about dislocations. In A533B steel without plastic deformation, for example, the magnetic-field amplitude less than $20 \mathrm{Oe}$ is sufficient. ${ }^{7}$ The sensitivity of the minor-loop method is also of interest in the present study.

\section{EXPERIMENTAL PROCEDURE}

We prepared samples of A533B steel, which are the same as those in Ref. 8. During the manufacturing process, the A533B steel was austenitized between 860 and $890^{\circ} \mathrm{C}$, followed by water quenching and tempering processes between 650 and $665^{\circ} \mathrm{C}$ for $139 \mathrm{~min}$. The grain size of the A533B steel samples determined by metallurgical microscopy is about $10 \mu \mathrm{m}$. Each sample was deformed in tension at room temperature in an Instron-type testing machine and was then cut into a doughnut shape, as shown in Fig. I. The magnetizing and detecting coils with 80 and 100 turns, respectively, were wound on these samples and the magnetization curves were measured at room temperature using a flux meter. It was ascertained that the measured results were in- 


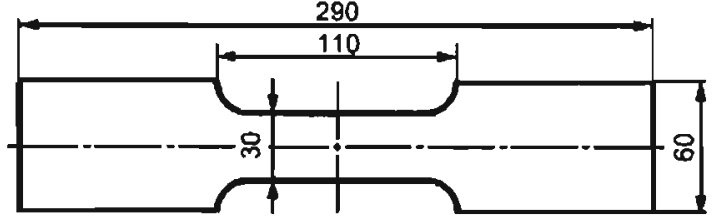

(a)

thickness $=5 \mathrm{~mm}$

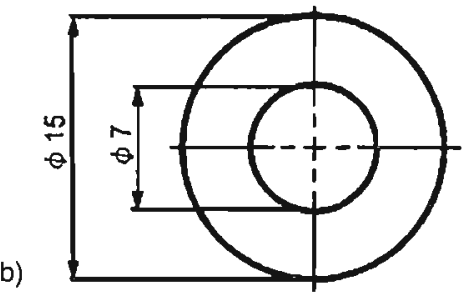

thickness $=5 \mathrm{~mm}$

FIG. 1. Size and shape of the samples, (a) for the tensile test and (b) for the measurements of minor hysteresis loops. The dimensions are in $\mathrm{mm}$.

dependent of the number of coil turns between 50 and 150 turns. The dislocations in each sample were observed in a transmission electron microscope (TEM).

\section{EXPERIMENTAL RESULTS}

The stress-strain curve of the A533B steel is shown in Fig. 2. The strain rate was $1.2 \% / \mathrm{min}$. The critical shear stress $\sigma_{C}$ is about $550 \mathrm{MPa}$ above which plastic deformation proceeds. The high value of $\sigma_{C}$ shows a high value of $\rho\left(\sim 3 \times 10^{10} \mathrm{~cm}^{-2}\right)$ in the initial state. ${ }^{8}$

The minor hysteresis loops were measured in the $H_{a}$ range from 0 to $50 \mathrm{Oe}$ and pseudomagnetic properties were obtained from every minor loop: ${ }^{6.7} M_{a}^{*}, H_{C}^{*}, M_{R}^{*}, W_{F}^{*}, \chi_{H}^{*}$, $\chi_{R}^{*}$, and $\chi_{a}^{*}$ corresponding to the saturation magnetization $M_{S}$, the coercive force $H_{C}$, the remanence $M_{R}$, the hysteresis loss $W_{F}$, and the susceptibilities, in the major loop, respectively. $W_{R}^{*}$ is the area enclosed by a minor loop in the second

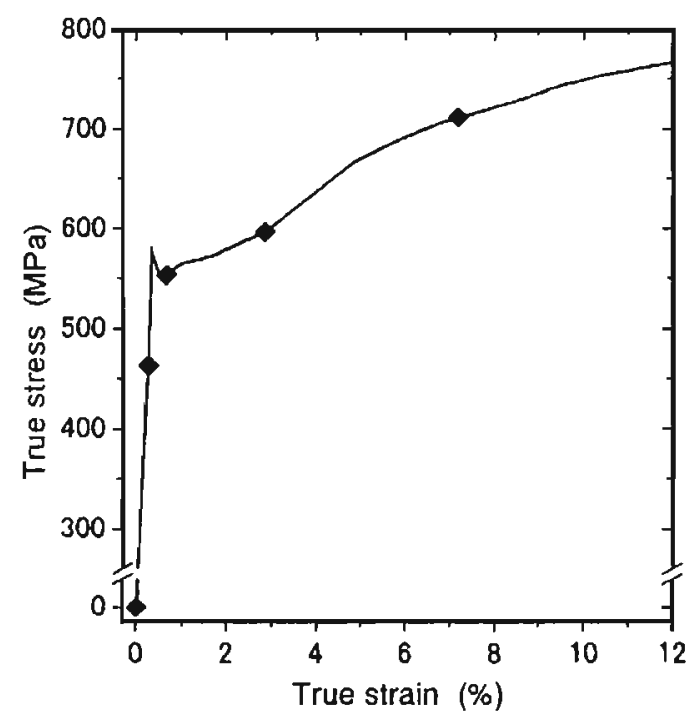

FIG. 2. Stress vs strain curve for A533B steel samples. The points mark the stresses at which magnetic measurements were taken.

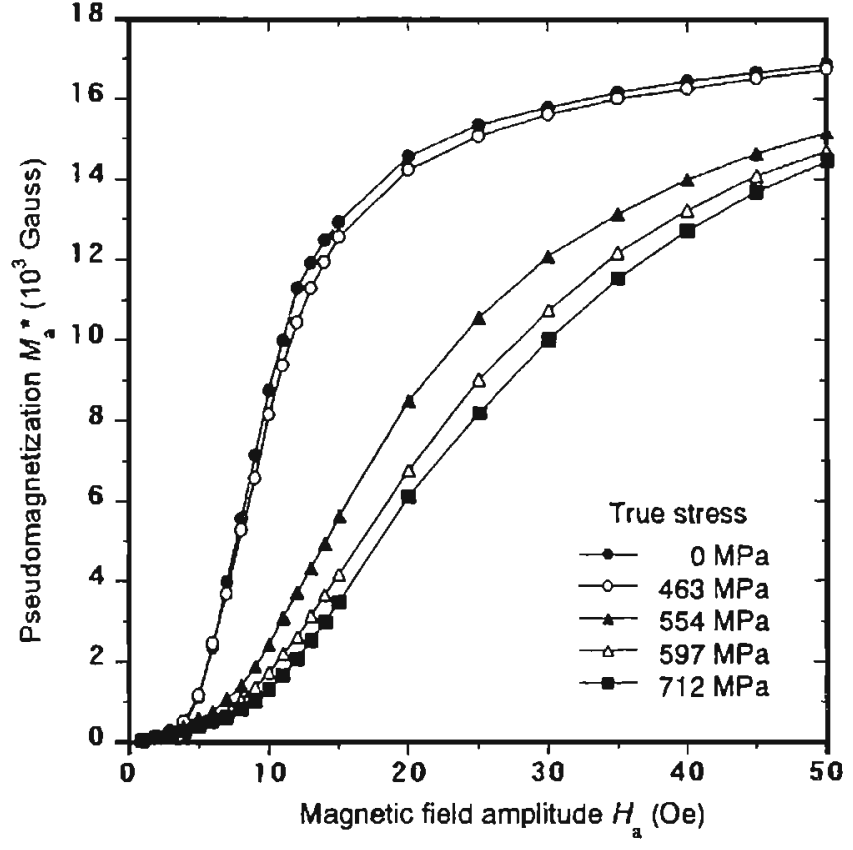

FIG. 3. Dependence of pseudomagnetization $M_{a}^{*}$ on the magnetic-field amplitude $H_{a}$ for A533B steel samples with tensile deformation.

quadrant. We can obtain information on the potential energy for domain-wall displacement by examining the relation between these pseudomagnetic properties. 6.7

Figure 3 shows the relation between pseudomagnetization $M_{a}^{*}$ and $H_{a}$ in A533B steel. The curves correspond to the virgin magnetization curves and give us the value of the initial susceptibility and the Rayleigh constant. While the curves are nearly independent of true stress below $\sigma_{C}$ $(\sim 550 \mathrm{MPa})$, they change considerably above $\sigma_{C}$ after plastic deformation.

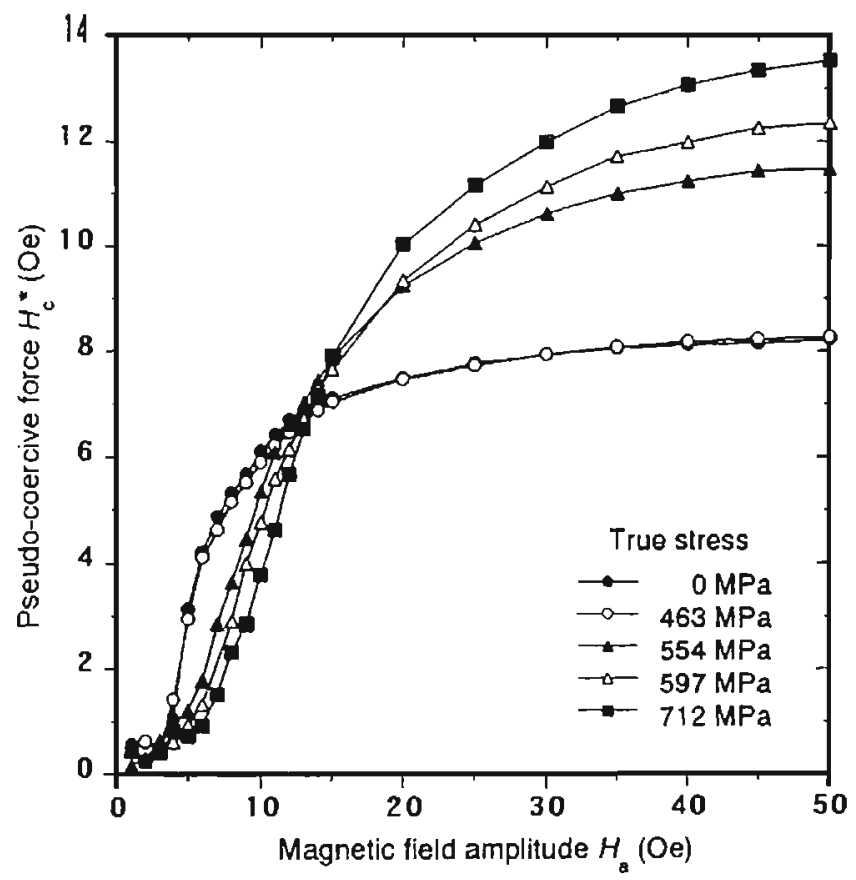

FIG. 4. Dependence of pseudocoercive force $H_{C}{ }^{*}$ on the magnetic-field amplitude $H_{a}$ for A533B steel samples with tensile deformation. 


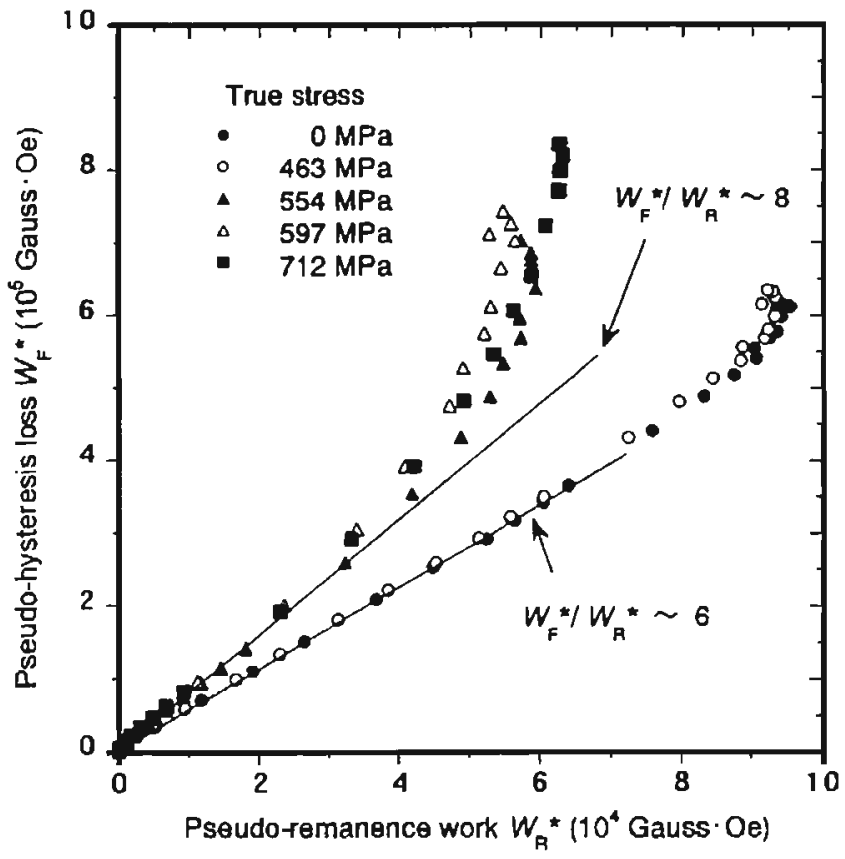

FIG. 5. The relation between pseudohysteresis loss $W_{F}{ }^{*}$ and pseudoremanence $W_{R}$ " for A533B steel samples with tensile deformation.

Figure 4 shows the $H_{a}$ dependence of pseudocoercive force $H_{C}{ }^{*}$. The value of $H_{C}{ }^{*}$ increases rapidly with the increase of $H_{a}$ in the second stage, the increase becomes small in the third stage, and $H_{C}{ }^{*}$ is finally saturated. The saturation of $H_{C} *$ occurs above 15 Oe before plastic deformation, while it does not even at 50 Oe for the samples with plastic deformation. The relation between $H_{C}$ and $H_{a}$ in each stage depends on the applied stress. The value of $H_{a}$ at which the third stage starts shifts to a larger field with increasing true stress.

The similarity of minor loops can be examined by com-

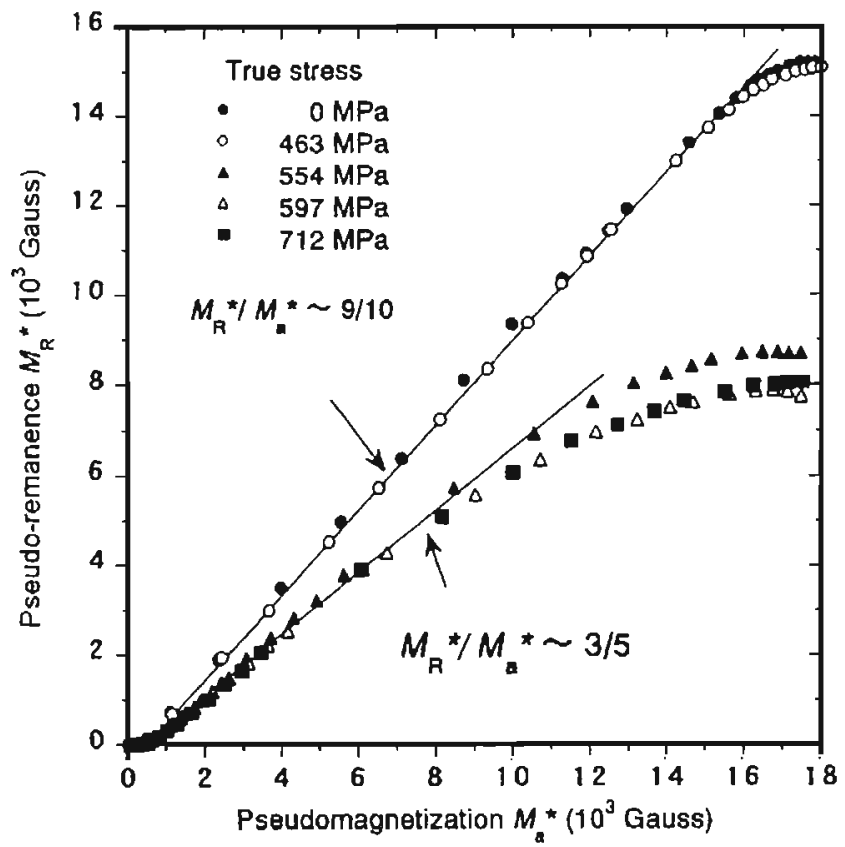

FIG. 6. The relation between pseudoremanence $M_{R}^{*}$ and pseudomagnetization $M_{a}^{*}$ for A533B steel samples with tensile deformation. paring $W_{R}^{*}$ with $W_{F}^{*}$ and $M_{R}^{*}$ with $M_{a}^{*}$ (see Figs. 5 and 6). The relation between $W_{R}^{*}$ and $W_{F}^{*}$ can be plotted on a straight line in the second stage except at large values, as shown in Fig. 5. The slope of the straight line is about 6.0 before plastic deformation and about 8.0 after plastic deformation in the second stage: $W_{R}{ }^{*} / W_{F}{ }^{*}=1 / 6$ and $1 / 8$ before and after plastic deformation, respectively. The deviation from the straight line at large $W_{R}{ }^{*}$ results from earlier saturation of $W_{R}^{*}$ than $W_{F}^{*}$. The saturation of $W_{R}^{*}$ can be explained in terms of the critical energy originating in the magnetostatic energy. ${ }^{5}$ The relation between $M_{a}{ }^{*}$ and $M_{R}{ }^{*}$ can be also plotted on a straight line in the second stage below $M_{R}{ }^{*}=5 \times 10^{3}$ and $1.5 \times 10^{4} \mathrm{G}$ for samples before and after plastic deformation, respectively, as shown in Fig. 6 . The gradual deviation of $M_{R}{ }^{*}$ from the straight line at large $M_{a}{ }^{*}$ is caused by the same origin, the critical energy. ${ }^{5}$ While the ratio of $M_{R}{ }^{*} / M_{a}^{*}$ is about $9 / 10$ before plastic deformation, it drastically changes to about $3 / 5$ due to plastic deformation and is independent of true stress. These experimental facts suggest that the minor hysteresis loops have a similar figure in the second stage before and after plastic deformation. This indicates the shape similarity of $180^{\circ}$ wall potential in the second stage.

The pseudohysteresis loss $W_{F}^{*}$ is the frictional energy of $180^{\circ}$ wall in one cycle of a minor loop and $M_{a}{ }^{*}$ corresponds to the potential energy at $H=H_{a}$ which is the highest energy in one minor-loop cycle. The pseudoremanence work $W_{R}^{*}$ is the work to displace the domain wall from the trough to the crest of the potential, and the pseudoremanence $M_{R}$ " corresponds to the trough of the potential. In our previous work for Fe single crystals, ${ }^{7}$ we found the relations between $W_{F}{ }^{*}$ and $M_{a}^{*}$ and between $W_{R}^{*}$ and $M_{R}^{*}$ in the second stage, which are represented, respectively, by

$$
W_{F}^{*}=W_{F}^{0}\left(\frac{M_{a}^{*}}{M_{S}}\right)^{n_{F}}
$$

and

$$
W_{R}^{*}=W_{R}^{0}\left(\frac{M_{R}^{*}}{M_{R}}\right)^{n_{R}} .
$$

Here, $W_{F}{ }^{0}$ and $W_{R}{ }^{0}$ are energy-loss and trough-depth coefficients, respectively. From least-squares fits using $M_{S}$ and $M_{R}$ obtained at $H_{a}=100 \mathrm{Oe}, n_{F}$ and $n_{R}$ were found to be $1.51 \pm 0.06$ and $1.42 \pm 0.03$, respectively, as shown in Figs. 7 and 8 . Such values are close to those obtained for Fe metal and its alloys $\left(n_{F}=n_{R}=1.5\right)$ reported previously, ${ }^{7}$ indicating that both $n_{F}$ and $n_{R}$ are nearly independent of kinds of materials and dislocation density. Assuming that $n_{F}=n_{R}=1.5$ for A533B steel, the dependence of $W_{F}^{0}$ and $W_{R}{ }^{0}$ on true stress was obtained, as shown in Fig. 9(a).

The relation between $H_{C}$ and $M_{R}$ " presents the height of crests in the potential and can be represented by

$$
H_{C}^{*}=H_{C}{ }^{0}\left(\frac{M_{R}^{*}}{M_{R}}\right)^{{ }^{\prime \prime} C},
$$

where $H_{C}{ }^{0}$ is a crest-height coefficient proportional to the potential height. ${ }^{7}$ As is seen in the least-squares fits in Fig. 10 , we found that while the value of $n_{C}$ is $0.33 \pm 0.03$ below 


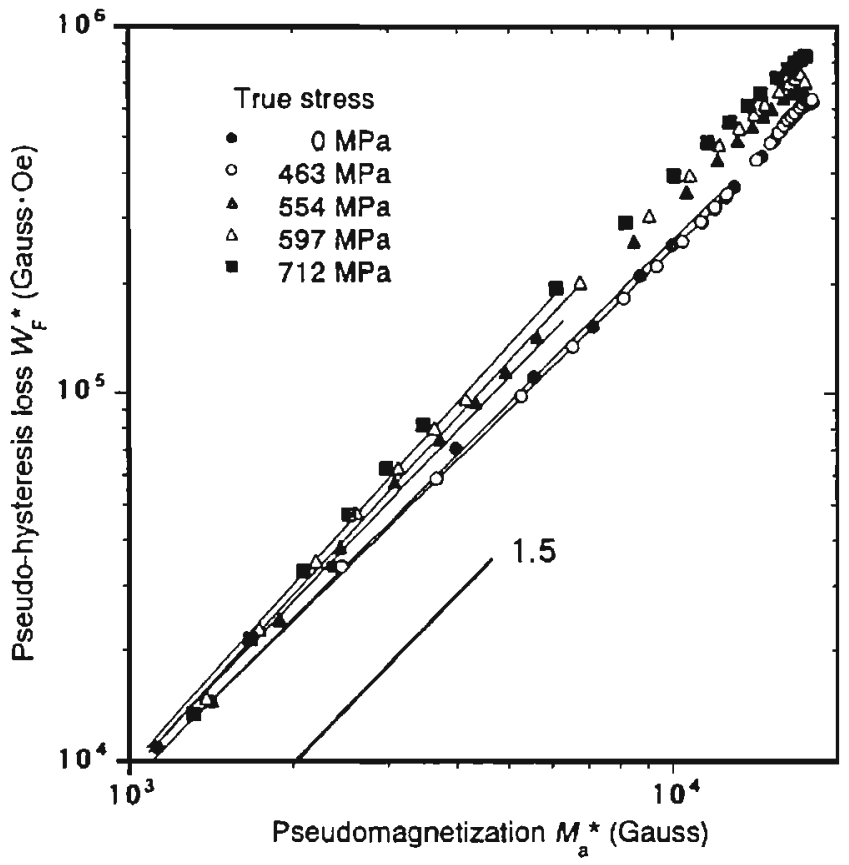

FIG. 7. The relation between pseudohysteresis loss $W_{F}{ }^{*}$ and pseudomagnetization $M_{a}{ }^{*}$ for A533B steel samples with tensile deformation in logarithmic scales. The solid lines denote the least-squares fits of the data in the second stage and the slope of 1.5 is shown for comparison with the observed slope.

$\sigma_{C}$, it changes to $0.50 \pm 0.04$ owing to plastic deformation and is independent of true stress above $\sigma_{C}$. The dependence of $H_{C}{ }^{0}$ on true stress is shown in Fig. 9(b).

The pseudosusceptibility at $H_{C}{ }^{*}, \chi_{H}{ }^{*}$, is a function of $H_{C}{ }^{*}$ and its reciprocal $R_{C}{ }^{*}\left(=1 / \chi_{H}^{*}\right)$ corresponds to the resistance for $180^{\circ}$ wall displacement at $H_{C}{ }^{*}$. As shown in Fig. 11 , both $R_{C}{ }^{*}$ and $H_{C}{ }^{*}$ have a simple relationship, in the second stage, given by

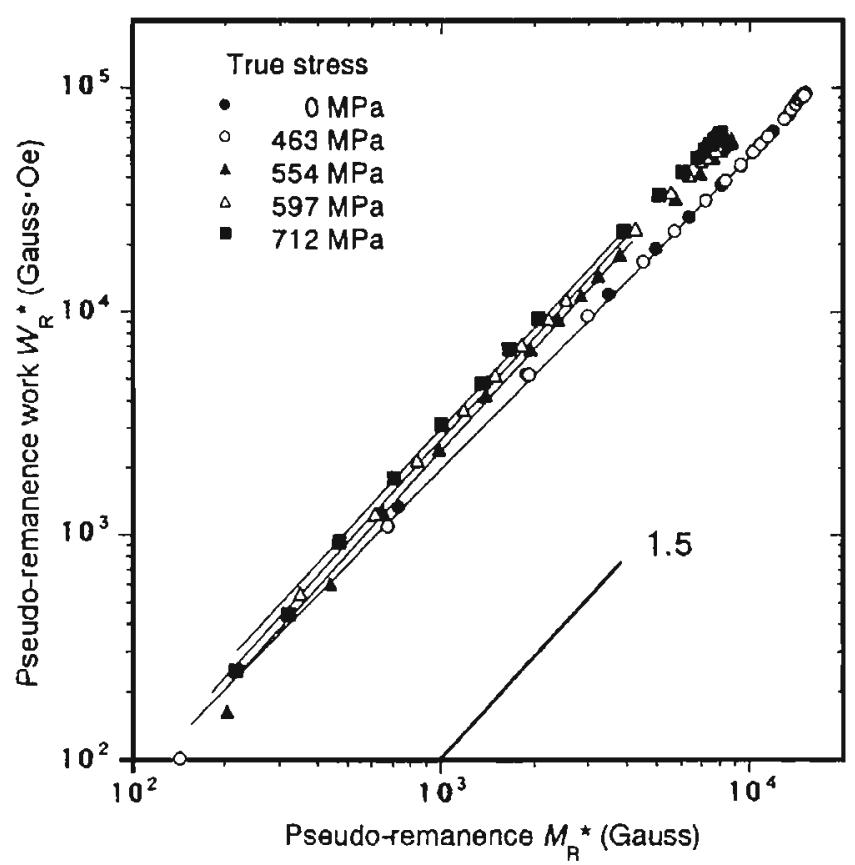

FIG. 8. The relation between pseudoremanence work $W_{R}{ }^{*}$ and pseudoremanence $M_{R}{ }^{*}$ for A533B steel samples with tensile deformation in logarithmic scales. The solid lines denote the least-squares fits of the data in the second stage and the slope of 1.5 is shown for comparison with the observed slope.

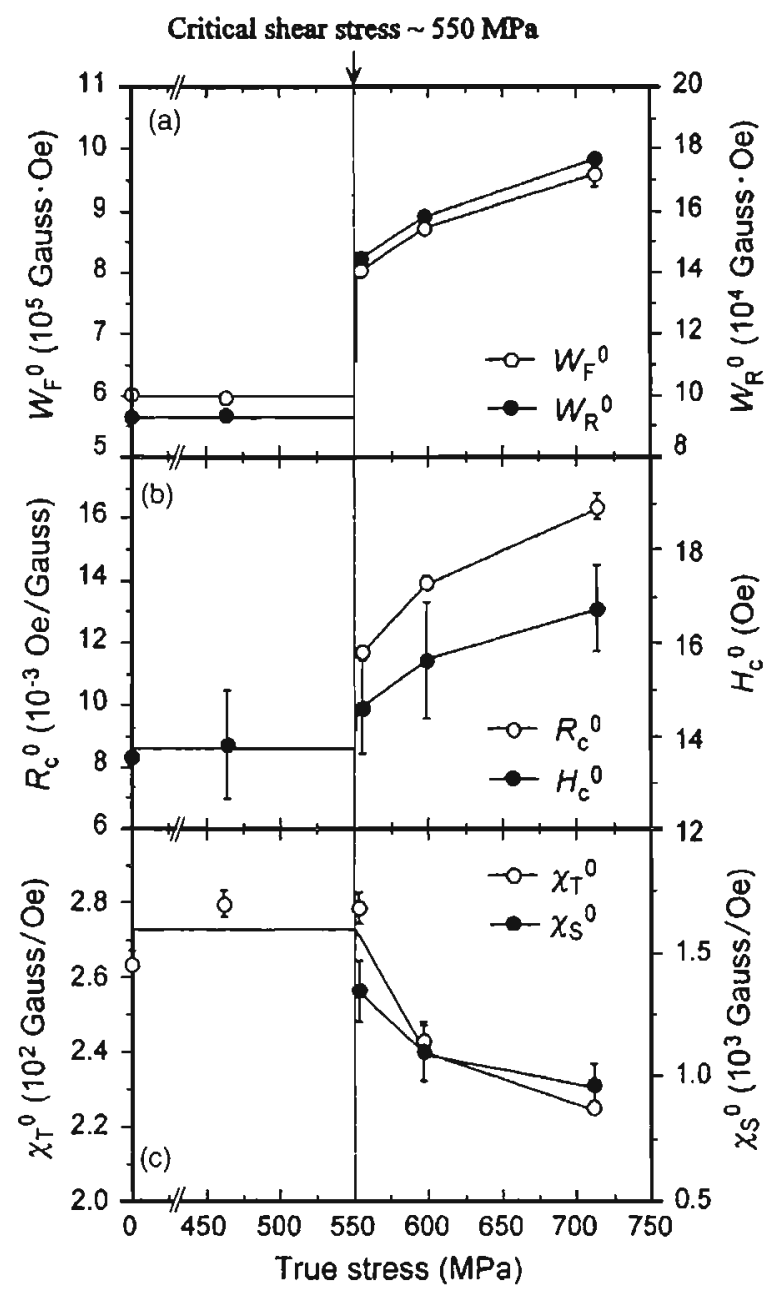

FIG. 9. True stress dependence of (a) $W_{F}{ }^{0}$ and $W_{R}{ }^{0}$, (b) $R_{C}{ }^{0}$ and $H_{C}{ }^{0}$, and (c) $\chi_{T}{ }^{0}$ and $\chi_{S}^{0}$ for A533B steel samples with tensile deformation.

$$
R_{C}^{*}=\frac{1}{\chi_{H}^{*}}=R_{C}^{0} \exp \left(-b \frac{H_{C}^{*}}{H_{C}}\right)
$$

where $R_{C}{ }^{0}$ is a resistance coefficient corresponding to the resistance at the potential crest, and $b$ is a resistance constant and depends on kinds of materials. ${ }^{7}$ The value of $b$ is $5.8 \pm 0.9$ below $\sigma_{C}$, but it abruptly reduces to $3.5 \pm 0.3$ and becomes almost constant above $\sigma_{C}$. The coefficient $R_{C}{ }^{0}$ steeply increases with increasing true stress, as shown in Fig. 9(b).

We can obtain information on $180^{\circ}$ wall displacement at the trough of the potential and on the average mobility of $180^{\circ}$ wall at the potential slope, from the relation between $\chi_{R}{ }^{*}$ and $M_{R}^{*}$ and between $\chi_{a}$ " and $M_{a}^{*}$, respectively. As shown in Figs. 12 and 13, in the second stage of magnetization process, the pseudosusceptibilities $\chi_{R}{ }^{*}$ and $\chi_{a}^{*}$ can be fitted by a simple formula, given by

$$
\chi_{R}^{*}=\chi_{T}^{0}\left(\frac{M_{R}^{*}}{M_{R}}\right)^{n_{T}}
$$




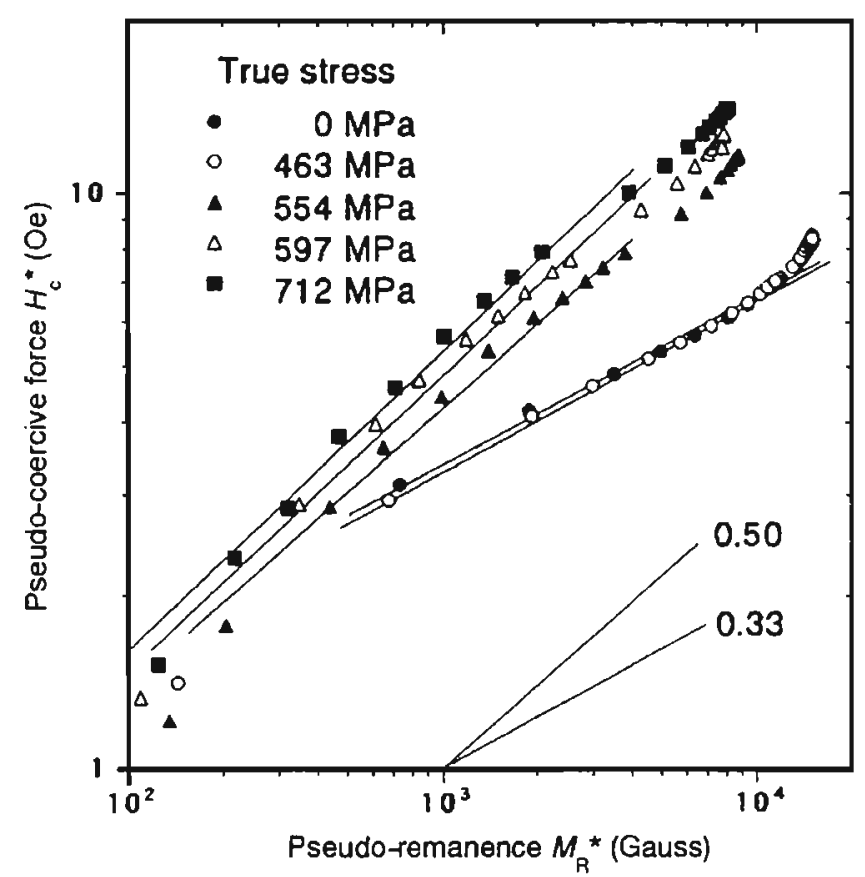

FIG. 10. The relation between pseudocoercive force $H_{C}{ }^{*}$ and pseudoremanence $M_{R}{ }^{*}$ for A533B steel samples with tensile deformation in logarithmic scales. The solid lines denote the least-squares fits of the data in the second stage and the slopes of 0.50 and 0.33 are shown for comparison with the observed slope.

$$
\chi_{a}^{*}=\chi_{S}^{0}\left(\frac{M_{a}^{*}}{M_{S}}\right)^{n_{S}}
$$

respectively. Here, $\chi_{T}{ }^{0}$ and $\chi_{S}{ }^{0}$ are mobility coefficients which are related with the mobility at the potential trough and the averaged mobility of the $180^{\circ}$ wall, respectively. $n_{T}$ and $n_{S}$ are constant and depend on kinds of materials. ${ }^{7}$ While

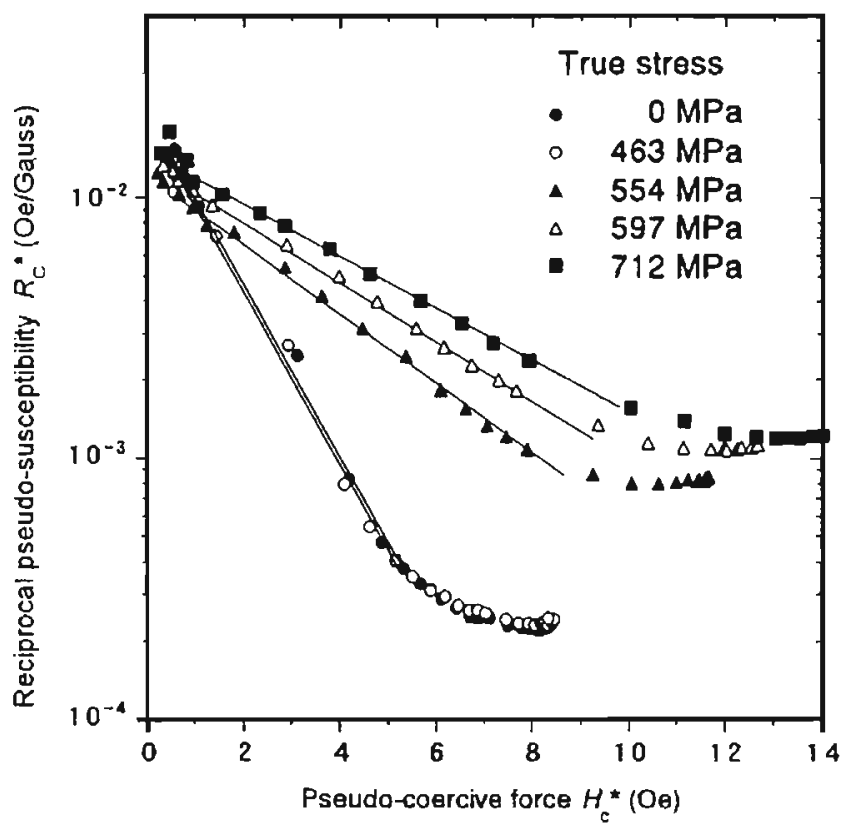

FIG. 11. The relation between reciprocal pseudosusceptibility $R_{C}{ }^{*}$ $\left(=1 / \chi_{H}{ }^{*}\right)$ and pseudocoercive force $H_{C}{ }^{*}$ for A533B steel samples with tensile deformation in logarithmic scales. The solid lines denote the leastsquares fits of the data in the second stage.

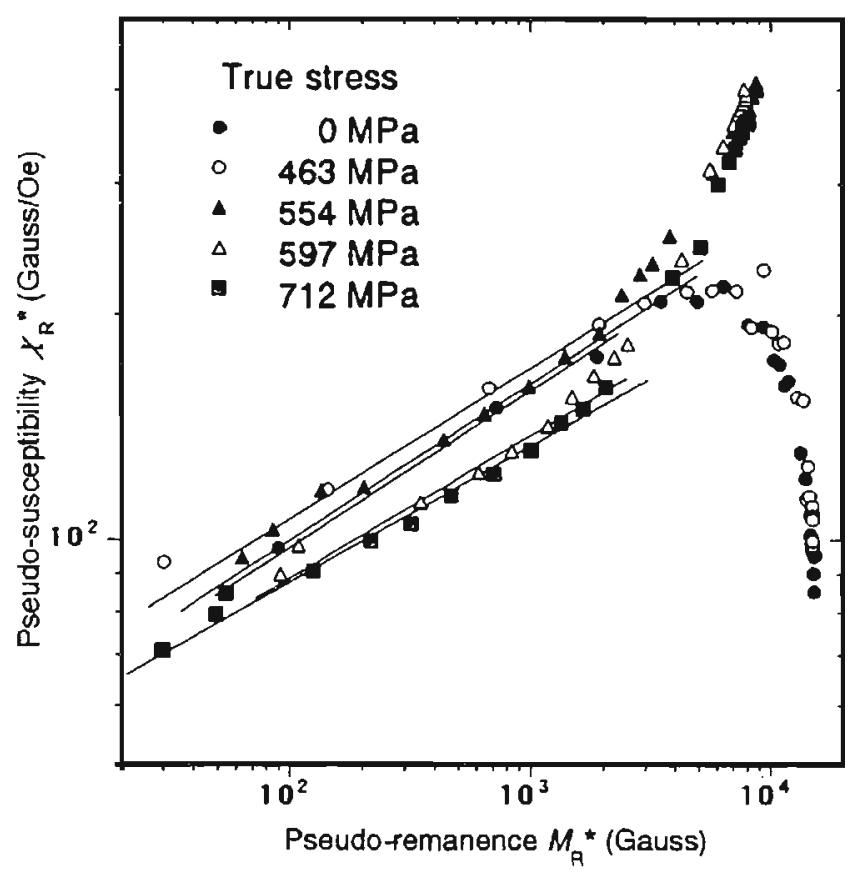

FIG. 12. The relation between pseudosusceptibility $\chi_{R}{ }^{*}$ and pseudoremanence $M_{R}{ }^{*}$ for A533B steel samples with tensile deformation. The solid lines denote the least-squares fits of the data in the second stage.

the value of $n_{T}$ is almost independent of true stress and is $0.19 \pm 0.03, n_{S}$ is $0.92 \pm 0.08$ and $0.58 \pm 0.06$ before and after plastic deformation, respectively. As shown in Fig. 9(c), both $\chi_{T}{ }^{0}$ and $\chi_{S}{ }^{0}$ decrease monotonically with increasing true stress above $\sigma_{C}$.

The influence of true stress on the $180^{\circ}$ wall potential and the mobility of the $180^{\circ}$ wall can be quantitatively evaluated from the magnetic coefficients $W_{F}^{0}, W_{R}{ }^{0}, H_{C}{ }^{0}, R_{C}{ }^{0}, \chi_{T}{ }^{0}$, and $\chi_{S}^{0}$. In view of residual internal stress, the true stress

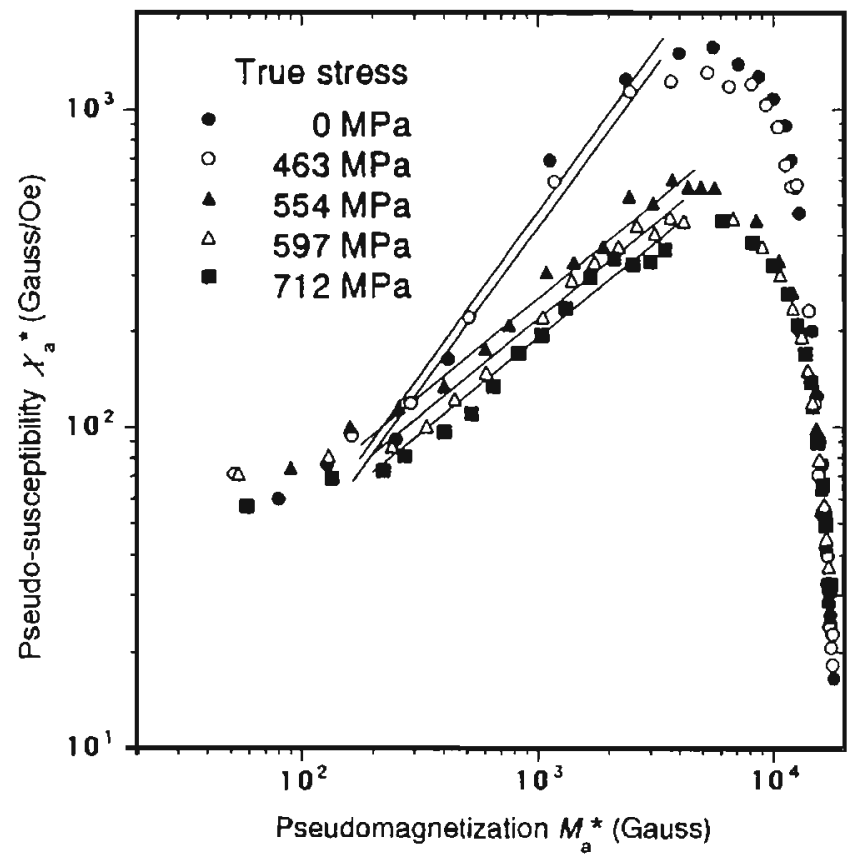

FIG. 13. The relation between pseudosusceptibility $x_{a}{ }^{*}$ and pseudomagnetization $M_{a}$ for A533B steel samples with tensile deformation. The solid lines denote the least-squares fits of the data in the second stage. 


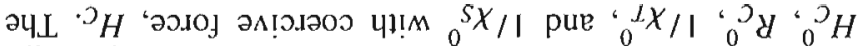

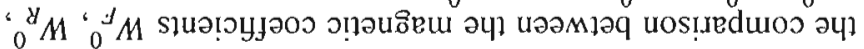
SMOYS $\downarrow[$ [anô!

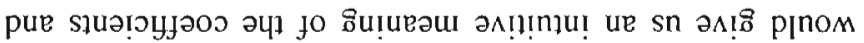

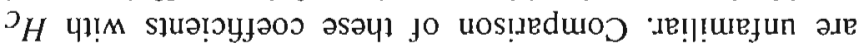

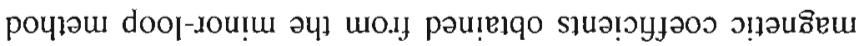

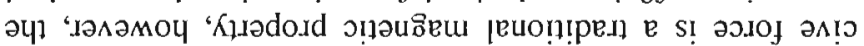
-Jว0ว t.

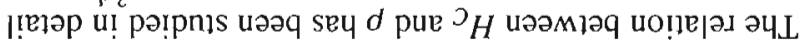

9 pue $\subseteq$ so̊! แ

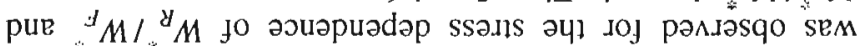

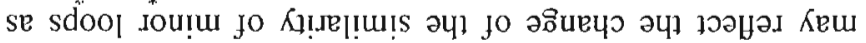

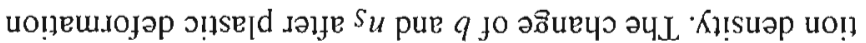

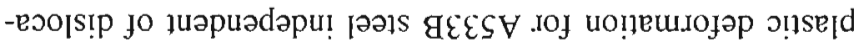

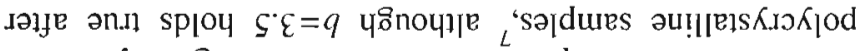

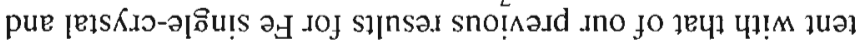

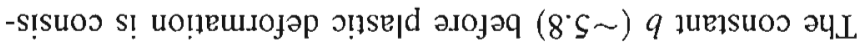

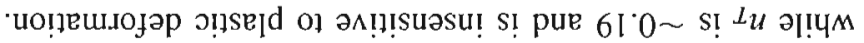

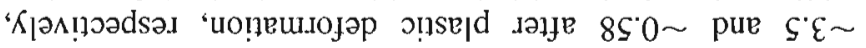

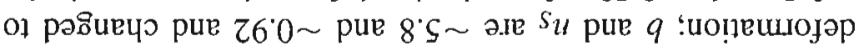

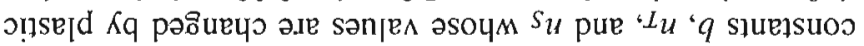

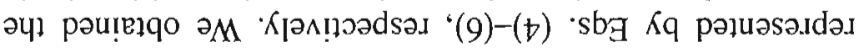

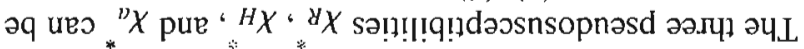

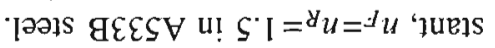

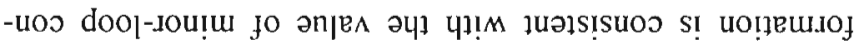

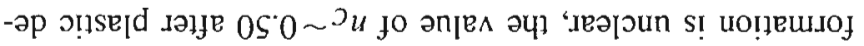

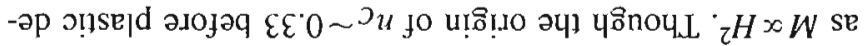

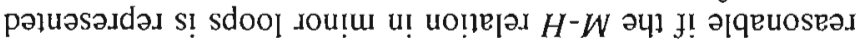

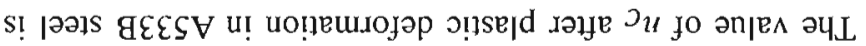

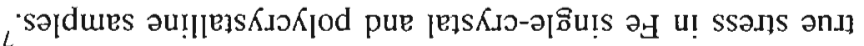

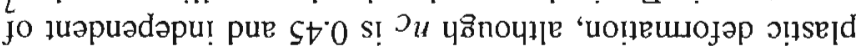

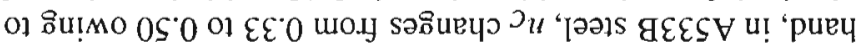

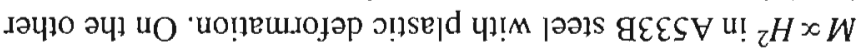

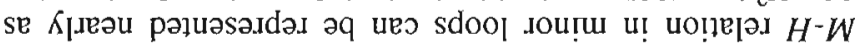

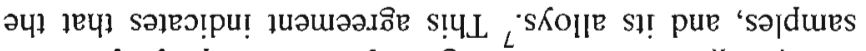

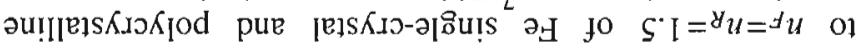

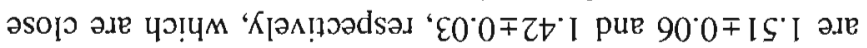

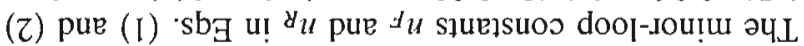

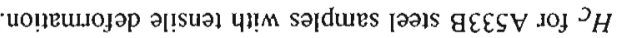

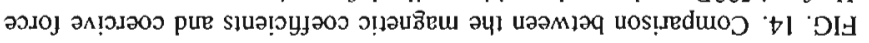

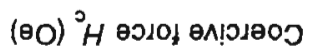

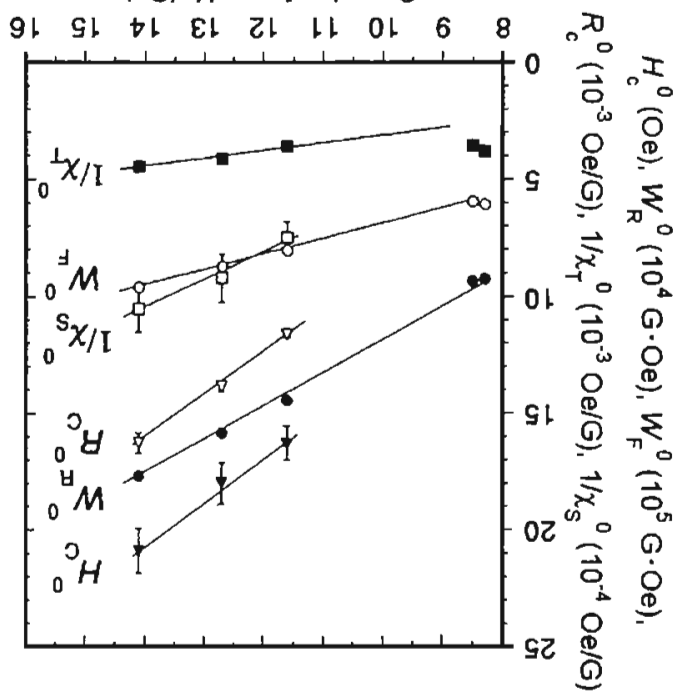

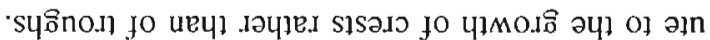
-q!.מuos p[nom uo!̣eujojap o!sejd $K q$ pasuespe pue paujo]

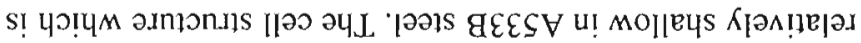

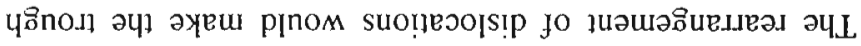
' ן

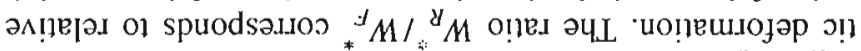

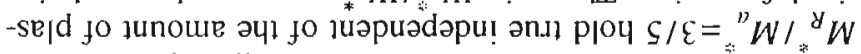

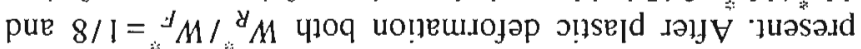

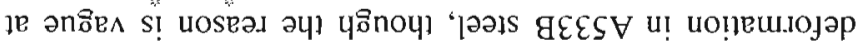

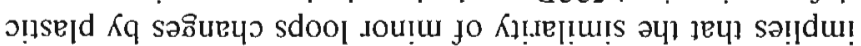

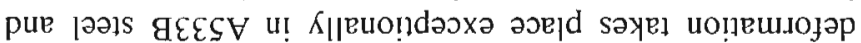

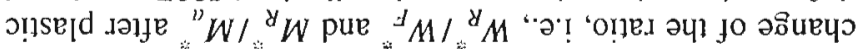

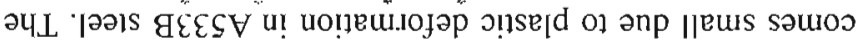

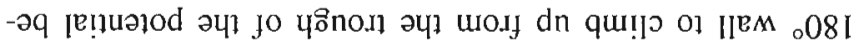

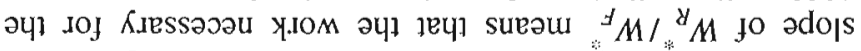

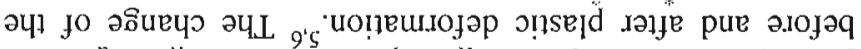

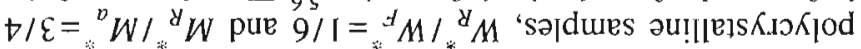

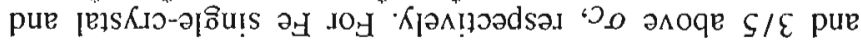

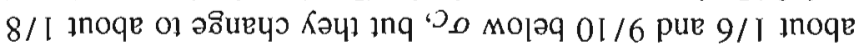

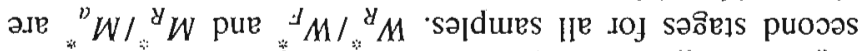

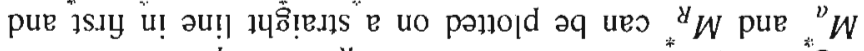

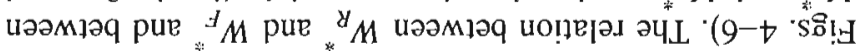

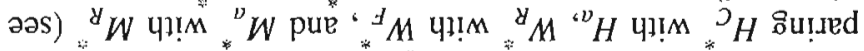

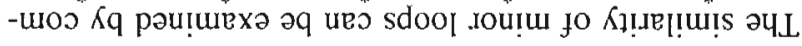

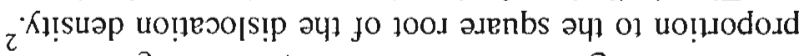

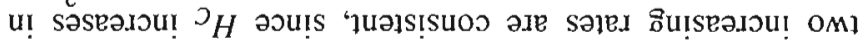

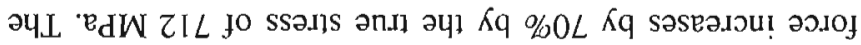

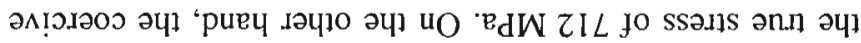

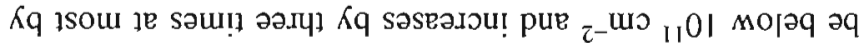

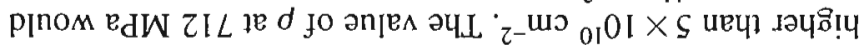

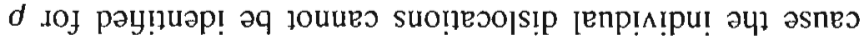

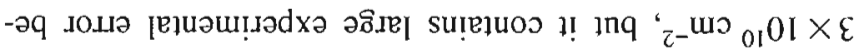
s! WäL Kq pəıеu!̣sə $d$ K!!

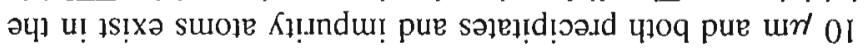
ınoqe s! วz!s u!̣.

NOISSnOSIO $\wedge \mathbf{I}$

- นolpeาวadxว

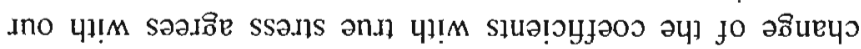

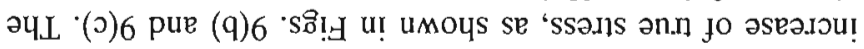

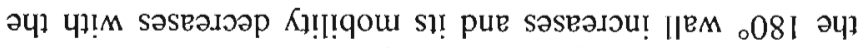

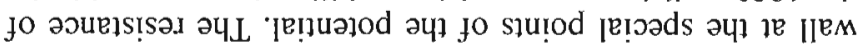
.08[ әपl jo K!!!!qou pue aoue]s!sar әul ol puodsan.jos ${ }_{0}{ }_{X}$

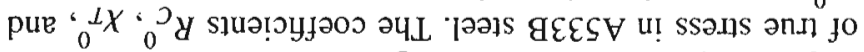

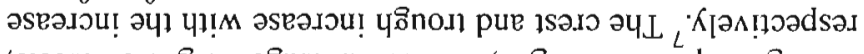

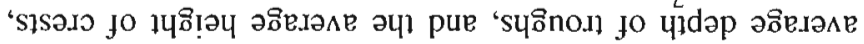

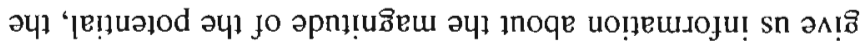

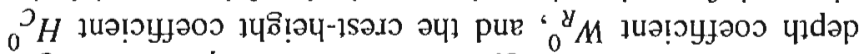

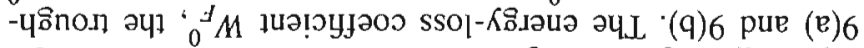

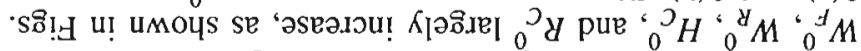

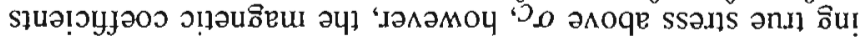

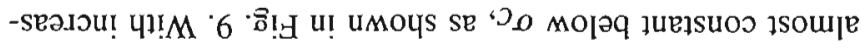

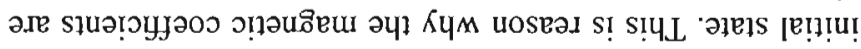

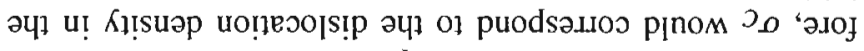

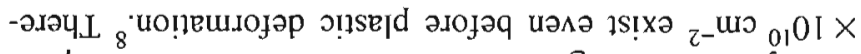

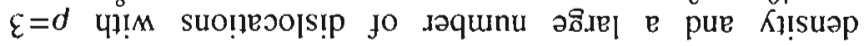

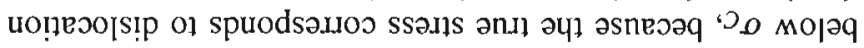

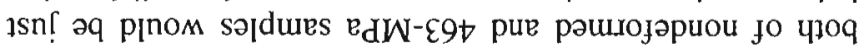


value of $H_{C}$ was obtained from a major loop with $H_{a}$ $=100 \mathrm{Oe}$ and both $\chi_{T}{ }^{0}$ and $\chi_{S}{ }^{0}$ are plotted by their reciprocal value. Simple proportional relations exist between the magnetic coefficients and $H_{C}$, since these magnetic properties were obtained by use of the magnetic domain wall as a sensor.

The dependence of $W_{R}{ }^{0}, W_{F}{ }^{0}$, and $H_{C}{ }^{0}$ on true stress is very similar to that of $H_{C}$. Both $W_{R}{ }^{0}$ and $H_{C}{ }^{0}$ increase at the same rate as $H_{C}$, but $W_{F}^{0}$ increases by $60 \%$ in the same range. These three coefficients would have the same relation with $\rho$ as $H_{C}$ in A533B steel. The small difference between the first two coefficients and the last would be attributed to the micro- and macrostructures of the potential due to the distribution of dislocations. $H_{C}{ }^{0}$ and $W_{R}{ }^{0}$ correspond to the height of crests and the depth of troughs, respectively, and they are more sensitive to the distribution of dislocations than $W_{F}^{0}$ in A533B steel. $R_{C}^{0}, 1 / \chi_{T}^{0}$, and $1 / \chi_{S}^{0}$ correspond to the resistance of the $180^{\circ}$ wall at the crest, the bottom of the trough, and the average slope of the potential, respectively. The mobility of the $180^{\circ}$ wall is sensitive to dislocations both at the crest and the average slope, and insensitive at the bottom of the trough (see Fig. 14). This mobility would be the characteristic of A533B steel and can be explained by the distribution of dislocations. The cell structure advances and the value of $\rho$ increases in the cell wall by plastic deformation in A533B steel. The increase of $\rho$ would mainly contribute to the macrostructure of potential, not to the microstructure.

The relations of Eqs. (4)-(6) deviate in comparison with those of Eqs. (1)-(3), though the former are more sensitive to dislocations than the later (see Figs. 7, 8, and 10-13). The deviation of the pseudosusceptibilities is their fundamental character. The pseudosusceptibilities depend on the mobility of the $180^{\circ}$ wall in the potential with a fractal structure. The mobility changes accidentally even at the bottom of trough, which seems to be the most stable point among three special points of the potential. The pseudoproperties $W_{F}^{*}, W_{R}^{*}$, and $H_{C}^{*}$ reflect the average structure of the potential and are insensitive to the fractal structure of the potential.

The coefficients give us much information on the internal structure including lattice defects and are useful for nondestructive evaluation of degradation in ferromagnetic materials. The coefficients have several advantages for nondestructive evaluation compared with the traditional parameters obtained from major loops. One of them is the small magnetic field required for measurements; the major loop can be obtained by the application of the magnetic field higher than 100 Oe in A533B steel, while the minor loops can be obtained by the magnetic field less than 20 Oe. This advantage is useful in the design of equipment. A set of minor loops yields us six magnetic coefficients and the information about the degradation is much greater than that available with major loops. The disadvantage of the minorloop method is the necessity of a large number of minor loops. It is possible, however, to decrease the number by taking advantage of the similarity of minor loops.

The initial susceptibility $\chi_{i}$ can be obtained from the virgin magnetization curves shown in Fig. 3. The value of $\chi_{i}$ contains more experimental errors than $H_{C}$. The magnetiza- tion does not increase linearly near the origin and it is difficult to draw a straight line at the origin. The nonlinearity near the origin is caused by the irreversible magnetization process in the initial stage. We could not observe the reversible magnetization process at the initial stage even in $\mathrm{Fe}$ single-crystal samples. ${ }^{5,6}$ The relation between $\chi_{i}$ and $\rho$ can be theoretically obtained on the supposition of the reversible process. ${ }^{4}$ Theoretically, Rayleigh constant $\alpha$ decreases in inverse proportion to $\rho{ }^{4}$ The reliable relation between $\alpha$ and true stress could not be obtained from the magnetization curves shown in Fig. 3. This can be due to the fact that the curves shown in Fig. 3 are not exactly the same as the virgin magnetization curves or the Rayleigh rule does not hold true in A533B steel. The experimental results of $\chi_{i}$ and $\alpha$ are not introduced in this paper.

Degradation proceeds by neutron irradiation in the nuclear pressure vessel. We have confirmed that magnetic coefficients obtained from minor loops are sensitive to degradation in A533B steel. The relation between the coefficients and the degradation by neutron irradiation will be reported in the near future.

\section{v. CONCLUSIONS}

The influence of dislocations on the $180^{\circ}$ wall displacement in A533B steel was examined experimentally by an analysis of minor hysteresis loops from the viewpoint of the potential. The following conclusive findings were forthcoming.

(1) The minor hysteresis loops are similar in the second stage independent of the magnetic-field amplitude, and there exist several simple relations between the pseudomagnetic properties: $W_{R}{ }^{*} / W_{F}^{*}=1 / 6$ and $1 / 8$ and $M_{R}^{*} / M_{a}^{*}=9 / 10$ and $3 / 5$ before and after plastic deformation, respectively.

(2) The magnetic coefficients $W_{F}{ }^{0}, W_{R}{ }^{0}$, and $H_{C}{ }^{0}$ yield information on the internal structure including lattice defects such as dislocations. With increasing dislocation density, both $W_{R}{ }^{0}$ and $H_{C}{ }^{0}$ largely increase, indicating that the height of crests and the depth of troughs increase with the height of the potential.

(3) The magnetic coefficients $R_{C}^{0}, \chi_{T}^{0}$, and $\chi_{S}^{0}$ yield information on $180^{\circ}$ wall mobility at the characteristic points of the potential. Among them, $R_{C}{ }^{0}$ and the reciprocal of $\chi_{S}{ }^{0}$ show a large increase with dislocation density. This means that the $180^{\circ}$ wall mobility is very sensitive to dislocations at the crest and the average slope of the potential.

(4) The magnetic-field amplitude required to obtain the magnetic coefficients is much lower than that for major loops and is less than 20 Oe for A533B steel with plastic deformation.

(5) The magnetic coefficients are effective for investigation of lattice defects, because they give much information about the potential of the $180^{\circ}$ wall and are more sensitive than the magnetic properties obtained from major 


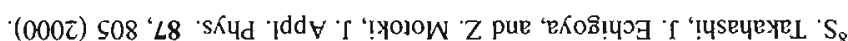

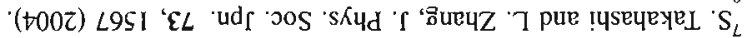

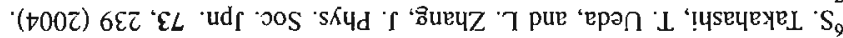
$\cdot(\varepsilon 00 z)$

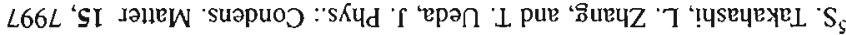

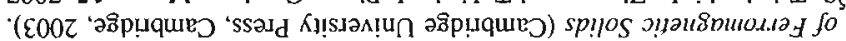

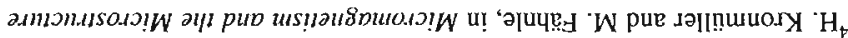

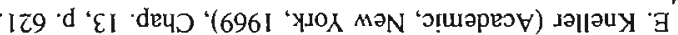

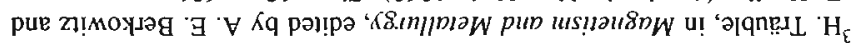

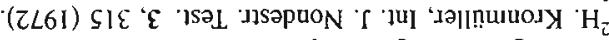

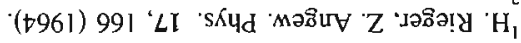

'osezOd aI mollə]

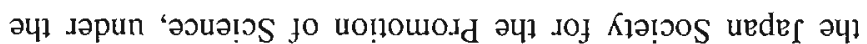

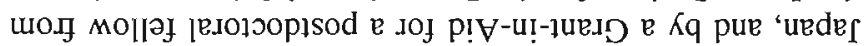

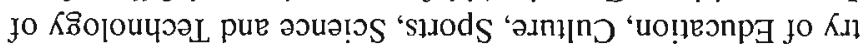

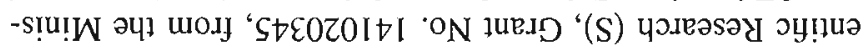

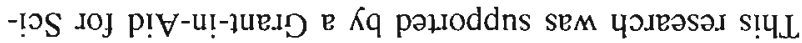

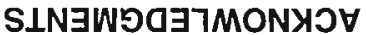

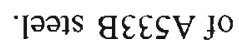

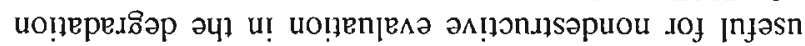
s! poylaur dool-rou!u әчl 'әлојәләчL ${ }^{2} H$ se yons sdoo 